\title{
The impact of adherence to HIV/ AIDS antiretroviral therapy on the development of drug resistance
}

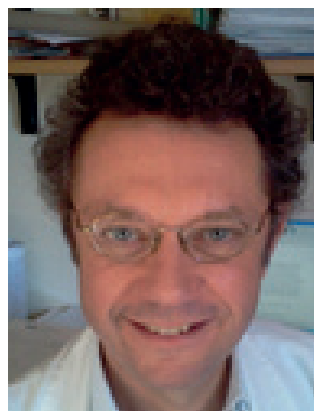

Stefano Rusconi*

\author{
"It is very clear that there is a distinct \\ relationship between the degree of adherence \\ to the antiretroviral medications and the \\ virologic suppression.”
}

First draft submitted: 5 February 2017; Accepted for publication: 9 February 2017; Published online: 28 April 2017

During the last decades, since 1996, there has been a strong commitment to reach both therapeutic efficacy and avoidance of drug resistance in case of virologic failure. The development of modern drug classes has facilitated the life of those involved in HIV/AIDS treatment, particularly HIV-infected subjects. There is still a gap between the perfect efficacy and the maximal adherence and tolerability, and of course all three aspects are interconnected.

It is very clear that there is a distinct relationship between the degree of adherence to the antiretroviral (ARV) medications and the virologic suppression. This was initially demonstrated by the ARV regimen during the first highly active ARV therapy (HAART) era and has been confirmed more recently with combination drugs, including non-nucleoside reverse-transcriptase inhibitors (NNRTI). The optimal cut-off is, again, the $95 \%$ adherence [1]. In both developing and developed countries there have been many demonstrations on how adherence affects clinical outcomes. Such an example comes from the the AIDS support organization initiative in Uganda: data show that, even within the first year and a half of ARV therapy, there is a significant difference, of approximately $40 \%$, in survival between adherent and nonadherent subjects [2]. In the MACS cohort [3], $\geq 80 \%$ suppression was observed with $80-84 \%$ adherence and the odds ratio for suppression versus $\geq 95 \%$ adherence was $1.43(0.61,3.33)$. In the ALIVE study [4], where $<35 \%$ were on newer drugs, only $71.4 \%$ were suppressed among those who reported $\geq 95 \%$ adherence. This suggests concerns related to nonadherence may be less of a barrier to the initiation of modern HAART regimens [5]. In the ICONA cohort, nonadherence was responsible for only $2 \%$ of treatment discontinuation in HIV-1 infected individuals starting their first-line ARV regimen after 2008 in Italy [6].

Nonadherence rates improve over time with the extension of follow-up visits, as shown by the CAPRISA initiative in South Africa. The nonadherence rate was $>40 \%$ at the initial visit and decreased to roughly $15 \%$ at visit 17 [7]. HAART adherence levels for individuals who started therapy in British Columbia increased over

*Infectious Diseases Unit, DIBIC Luigi Sacco, University of Milan, Via G.B. Grassi 74, 20157 Milan, Italy; Tel.: +390250319761; Fax: +390250316758; stefano.rusconi@unimi.it

\section{KEYWORDS}

$\bullet$ adherance $\bullet$ drug resistance $\bullet$ HIV 
"There is a tight interplay between adherence and virological suppression, and drug resistance..." time during 1996-2012. While only 37\% of individuals had adherence levels $\geq 95 \%$ in 1996 , this increased to $71 \%$ for individuals ( $\mathrm{p}=0.0032$ ) by 2012 [8]. There is a tight interplay between adherence and virological suppression, and drug resistance, as shown by the work of Nachega $e t a l$. Lower pill burden was significantly associated with both better adherence and virological suppression in 19 studies including 6312 adult patients [9]. Likely due to safer and more effective drugs and an improvement in patient's adherence, recent data dealing with the epidemiology of resistance to ARVs showed a declining prevalence of HIV-1 drug resistance in experienced individuals in Western Europe (SEHERE collaboration) with the exception of NNRTI [10]. The proportion with extensive three-class resistance over calendar years by the last and the cumulative genotype decreased between 2005 and 2008 and the proportion of cases with an exhaustion of drug options by the last and the cumulative genotype was $<5 \%$ in 2008 [10].

When we think about drug resistance, the paradigmatic view ranges from an easy probability of developing drug resistance, detected via minority variants methods, id est, resistance toward NNRTI and the extent of cross-resistance to newer compounds. An example of the first dynamic is the low frequency of NNRTI mutations K103N, Y181C and NRTI mutations M184V, K65R, and the risk of NNRTI-based ARV treatment failure. Minority drug-resistant variants were found in $14 \%$ of samples in this systematic review [11]. The second example is illustrated by the resistance to the newest ARV class, which is well established in the clinical arena: the integrase inhibitors (INI). A recent report from the USA underlines that one out of five patients tested during the first 3 years of the availability of a genotypic resistance test for INI harbored at least one major integrase-resistance mutation [12]. The positive aspect of the resistance to INI is that cross-resistance between raltegravir, or elvitegravir, and dolutegravir is very contained in raltegravirnaive patients. In raltegravir-pretreated patients, $50(41.7 \%)$ of the integrase sequences obtained at failure of raltegravir therapy did not contain any mutation at codons 143, 148 and 155 [13].

A totally different story may be told in the upcoming scenario of long-acting (LA) compounds, that is, cabotegravir and rilpivirine that can be administered via a subcutaneous or intramuscular injection. These compounds possess a very long half-life, thus can resolve most of the problems connected with adherence. But there are still missing spots: we have no data regarding the virologic protection after the final injection of these compounds. If a patient withdraws from a trial, will the pharmacological 'tail' still be protective or pose a serious threat for the development of drug resistance? A single case of an emergent NNRTI-resistant virus has been reported in a subject from a rilpivirine LA $300 \mathrm{mg}$ Phase I tissue pharmacokinetic study [14]. The LA pharmacokinetics may not be sufficient in nonadherent patients and consequences of this insufficient drug exposure could appear later on, without being adjustable thus jeopardizing patient's therapeutics [15].

Thus, there is still an ongoing debate on situations when the maximal virologic efficacy is essential and maximizing drug adherence is a priority. In the near future, more friendly drug regimens/administration devices will help in maintaining optimal therapeutic results and keeping regimen forgiveness at the highest level.

\section{Financial \& competing interests disclosure}

The author has no relevant affiliations or financial involvement with any organization or entity with a financial interest in or financial conflict with the subject matter or materials discussed in the manuscript. This includes employment, consultancies, honoraria, stock ownership or options, expert testimony, grants or patents received or pending, or royalties.

No writing assistance was utilized in the production of this manuscript.

\section{References}

1 Nachega JB, Hislop M, Dowdy DW, Chaisson RE, Regensberg L, Maartens G. Adherence to nonnucleoside reverse transcriptase inhibitor-based HIV therapy and virologic outcomes. Ann. Intern. Med. 146, 564-573 (2007).

2

Abaasa AM, Todd J, Ekoru K et al. Good adherence to HAART and improved survival in a community HIV/AIDS treatment and care programme: the experience of The AIDS Support Organization (TASO), Kampala, Uganda. BMC Health Serv. Res. 8, 241 (2008).

, Jacobson L, Detels R et al. Acquired immune deficiency syndrome occurring within 5 years of infection with human immunodeficiency virus type-1: the Multicenter AIDS Cohort Study. J. Acquir. Immune Defic. Syndr. 5, 490-496 (1992).
4 Vlahov D, Anthony JC, Munoz A et al. The ALIVE study, a longitudinal study of HIV-1 infection in intravenous drug users: description of methods and characteristics of participants. NIDA Res. Monogr. 109, 75-100 (1991).

5 Viswanathan S, Detels R, Mehta SH, Macatangay BJC, Kirk GD, Jacobson LP. Level of adherence and HIV RNA suppression in the current era of highly active 
antiretroviral therapy (HAART). AIDS Behav. 19, 601-611 (2015).

Di Biagio A, Cozzi-Lepri A, Prinapori R et al. Discontinuation of initial antiretroviral therapy in clinical practice: moving toward individualized therapy. J. Acquir. Immune Defic. Syndr. 71, 263-271 (2016).

7 Maqutu D, Zewotir T. Optimal HAART adherence over time and time interval between successive visits: their association and determinants. AIDS Care 23, 1417-1424 (2011).

8 Montaner JSG, Lima VD, Harrigan PR et al. Expansion of HAART coverage is associated with sustained decreases in HIV/AIDS morbidity, mortality and HIV transmission: the "HIV Treatment as Prevention" experience in a Canadian setting. PLoS ONE 9(2), e87872 (2014).
9 Nachega JB, Parienti JJ, Uthman OA et al. Lower pill burden and once-daily antiretroviral treatment regimens for HIV infection: a meta-analysis of randomized controlled trials. Clin. Infect. Dis. 58, 1297-1307 (2014).

10 De Luca A, Dunn D, Zazzi M et al. Declining prevalence of HIV-1 drug resistance in antiretroviral treatment-exposed individuals in Western Europe. J. Infect. Dis. 207, 1216-1220 (2013).

11 Li JZ, Paredes R, Ribaudo HJ et al. Low-frequency HIV-1 drug resistance mutations and rick of NNRTI-base antiretroviral treatment failure: a systematic review and pooled analysis. JAMA 305, 1327-1335 (2011).

12 Hurt CB, Sebastian J, Hicks CB, Eron JJ. Resistance to HIV integrase strand transfer inhibitors among clinical specimens in the United States, 2009-2012. Clin. Infect. Dis. 58, 423-431 (2014).

13 Saladini F, Meini G, Bianco C et al. Prevalence of HIV-1 integrase mutations related to resistance to dolutegravir in raltegravir naïve and pretreated patients. Clin. Microbiol. Infect. 18, E428-E430 (2012).

14 Penrose KJ, Parikh UM, Hamanishi KA et al. Selection of rilpivirine-resistant HIV-1 in a seroconverter from the SSAT 040 trial who received the $300 \mathrm{mg}$ dose of long-acting rilpivirine (TMC278LA). J. Infect. Dis. 213, 1013-1017 (2016).

15 Hickey MB, Merisko-Liversidge E, Remenar JF, Namchuk M. Delivery of long-acting injectable antivirals: best approaches and recent advances. Curr. Opin. Infect. Dis. 28, 603-610 (2015). 